\title{
Pediatrics Cushing's Disease: a diagnostic challenge
}

Ma Joana Santos ${ }^{1}$, Sofia Martins ${ }^{2,4}$, Ana Antunes ${ }^{2,4}$, Rui Almeida ${ }^{3,5}$, Olinda Marques ${ }^{1,4,5}$

1. Endocrinology Department; 2. Paediatrics Department; 3. Neurosurgery Department; 4. Paediatric Endocrine

Group; 5. Pituitary Tumours Group, Hospital de Braga, Braga, Portugal

Introduction: Cushing's disease $(C D)$ is rare in children. It's most common clinical manifestations are growth retardation, changes in pubertal development and weight gain. The diagnosis, based on clinical suspicion, is often hampered by the non identification of the microadenoma in MRI.

\section{Clinical case}

- History of present illness: Fourteen year old boy observed in a pediatric endocrinology consultation due to increased weight gain since age of 9 and short stature and growth arrest (growth velocity < P3) since age of 12.

-Past Medical History: irrelevant

-Family history: overweight in both parents; one 18 year old brother with normal growth and pubertal development

\section{- Physical Exam}

- Moon face, facial flushing, acne, abdominal obesity.

-BP 131/75mmHg (P95), Weight 54.5Kg (P75), height $135 \mathrm{~cm}$ (<<<P3), BMI 29.9Kg/m2 (>P95)

-Tanner: P2, A1, G1.

- Similar bone and chronological ages.

\section{Investigation}

\begin{tabular}{|l|l|}
\hline $\begin{array}{l}\text { Free urinary cortisol } \\
\text { 24h (ug/day) (N:20- } \\
\text { 90) }\end{array}$ & $\begin{array}{l}734(\uparrow 8 \mathrm{x}) \\
257(\uparrow 2,8 \mathrm{x})\end{array}$ \\
\hline $\begin{array}{l}\text { Cortisol (1mg } \\
\text { dexamethasone test) }\end{array}$ & $\mathbf{7 . 6}$ (个1,8x) \\
\hline $\begin{array}{l}\text { Rythm (8h/ 23H) } \\
\text { ACTH } \\
\text { Cortisol }\end{array}$ & $\begin{array}{l}35,8 / 18,7 \mathrm{pg} / \mathrm{ml} \\
17,1 / 9,74 \mathrm{ug} / \mathrm{dl}\end{array}$ \\
\hline \multicolumn{2}{|l|}{ ACTH dependent hypercortisolism } \\
\hline
\end{tabular}

\begin{tabular}{|cc|}
\hline $\begin{array}{c}\text { VIGILANCE WHILE } \\
\text { UNCERTAIN } \\
\text { DIAGNOSIS }\end{array}$ & $\begin{array}{c}\text { INFERIOR PETROUS } \\
\text { SINUS CATHETERIZATION }\end{array}$ \\
$\begin{array}{c}\text { - Risks of the procedures } \\
\text { - Few experience in our } \\
\text { centre at that time }\end{array}$ & $\begin{array}{l}\text { AND/OR SURGERY } \\
\text { - Cushing's complications } \\
\text { - Growth and pubertal } \\
\text { development delay }\end{array}$ \\
\hline
\end{tabular}

Total cholesterol 196, HDL-C 69, TG 56 mg/dl FT3 $4.99 \mathrm{pg} / \mathrm{ml}$, FT4 $1.7 \mathrm{ng} / \mathrm{dl}$, TSH $2.9 \mathrm{uUI} / \mathrm{ml}$ GH $1.57 \mathrm{ng} / \mathrm{ml}$, IGF1 300ng/ml, IGFBP3 $6 \mathrm{mg} / \mathrm{L}$

PRL 530 (<360uUl/ml)

$\mathrm{FSH} 0.33 \mathrm{mlU} / \mathrm{ml}$, LH $<0,1 \mathrm{mlU} / \mathrm{ml}$ Total testost 37,26ng/dl (Tanner 2) Estradiol: $1.55 \mathrm{ng} / \mathrm{dl}$ (Tanner 2) Androstenedione: $1.2 \mathrm{ng} / \mathrm{ml}$ (Tanner 2) DHEAS: $244 \mathrm{ng} / \mathrm{dl}$ (Tanner 2) Insulin 22uUI/ml, C Pep $1.94 \mathrm{ng} / \mathrm{ml}$

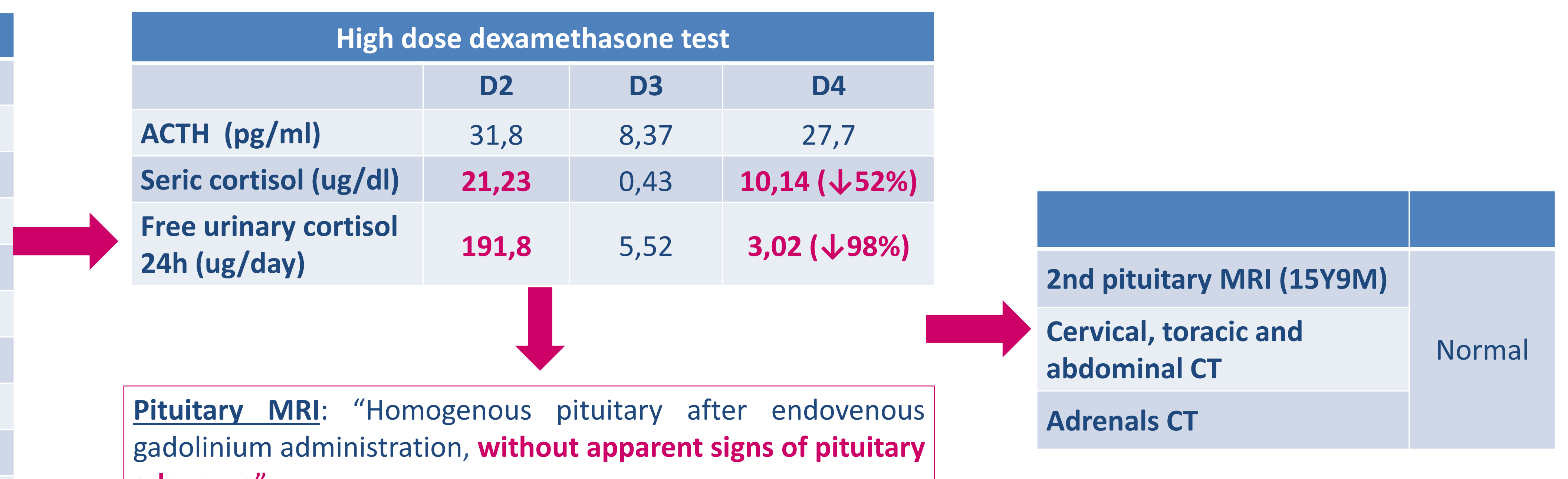
adenoma"

\begin{tabular}{|l|c|c|c|}
\multicolumn{4}{|c|}{ Inferior Petrous Sinus Catheterization } \\
\hline ACTH & Right side & Left side & Peripheric \\
\hline $\mathbf{- 1 5 ^ { \prime }}$ & $>\mathbf{1 2 5 0}$ & $\mathbf{6 2 , 0}$ & 36,3 \\
\hline $\mathbf{0}^{\prime}$ & 203 & 54,8 & 40,3 \\
\hline $\mathbf{2}^{\prime}$ & $\mathbf{1 2 0 1}$ & $\mathbf{2 4 5 , 0}$ & $\mathbf{4 3 , 8}$ \\
\hline $\mathbf{5}^{\prime}$ & 50,8 & $\mathbf{7 9 , 2}$ & 46,8 \\
\hline $\mathbf{1 0}$ & 86,0 & 230,0 & 52,8 \\
\hline $\mathbf{1 5}$ & 80,0 & 84,9 & 57,2 \\
\hline
\end{tabular}

Ratio Central/Peripheric $>\mathbf{2}$ Ratio Right/Left $>1.4$

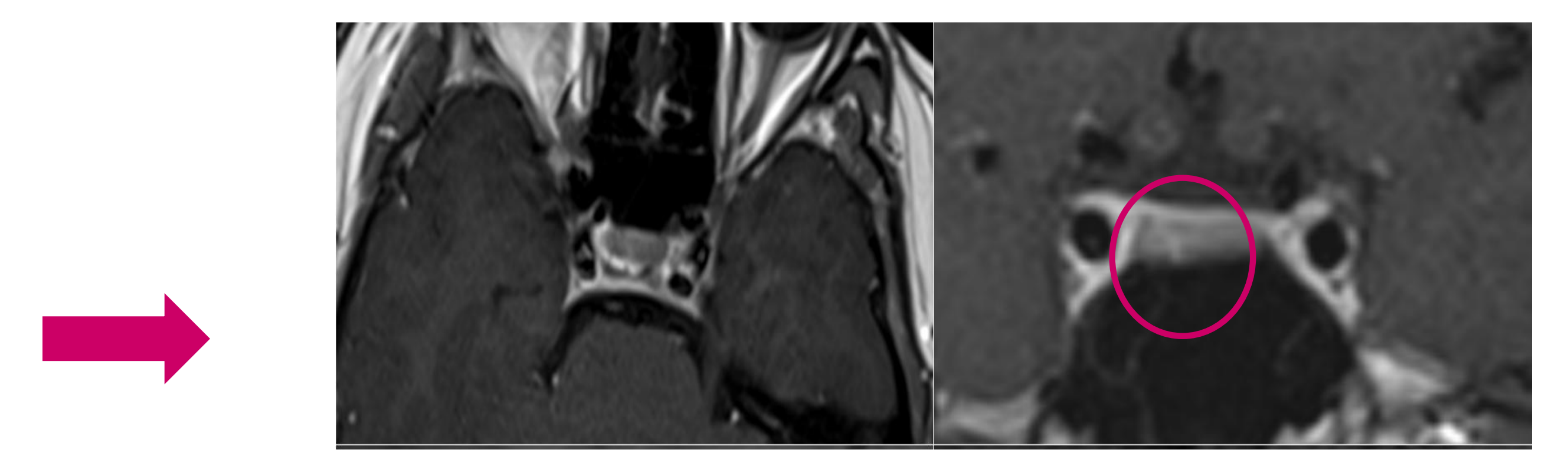

3rd pituitary MRI: "Discrete asymmetry of the anterior pituitary, slightly higher on the right side; adjacent to the supero-medial wall of the cavernous sinus, there is a hyperintense area on T2 with $2.5 \mathrm{~mm}$, with a slight uptake of gadolinium in the dynamic study"

\section{Treatment and evolution}

\begin{tabular}{|c|c|c|c|}
\hline \multicolumn{2}{|c|}{15 years and 7 months } & \multicolumn{2}{|c|}{ Follow-up: 19 years (3Y 4M after surgery) } \\
\hline \multirow{2}{*}{ Transfenoidal surgery } & \multirow{2}{*}{$\begin{array}{l}\text { Apparent macroscopic tumor } \\
\text { removal }\end{array}$} & Weight & 59 Kg (P10-25) \\
\hline & & Height & $151 \mathrm{~cm}(<<P 3)$ \\
\hline \multirow{2}{*}{ Seric cortisol after surgery } & \multirow{2}{*}{$0,32 \mathrm{ug} / \mathrm{dl}$ (<1,8ug/dl) } & BMI & $25.9 \mathrm{Kg} / \mathrm{m} 2$ (P75-90) \\
\hline & & BP & $112 / 47 \mathrm{mmHg}$ \\
\hline Histology & No pituitary adenoma identified & Tanner & V (Test 20cc bilateral) \\
\hline \multirow{2}{*}{ Hydrocortisone post surgery } & \multirow{2}{*}{ Suspended only 10 months after } & Bone vs chronological age & Similar (19 years) \\
\hline & & ACTH & $22 \mathrm{pg} / \mathrm{ml}(\mathrm{N}<46)$ \\
\hline \multirow[t]{4}{*}{ Evolution } & \multirow{4}{*}{$\begin{array}{l}\text { Clinical and analytical } \\
\text { improvement with increased } \\
\text { growth velocity and normal } \\
\text { pubertal development }\end{array}$} & Seric cortisol & 14,7 ug/dl $(4,3-22,4)$ \\
\hline & & Free urinary cortisol & $271,2 \mathrm{ug} / 24 \mathrm{~h}(55,5-286)$ \\
\hline & & IGF1 & $332,3 \mathrm{ng} / \mathrm{ml}(247,3-481,7)$ \\
\hline & & Total testosterone & $559,2 \mathrm{ng} / \mathrm{dl}(241-827)$ \\
\hline
\end{tabular}

Conclusion: This case had a successful evolution, but highlights the difficulties of CD diagnosis in children. Time between clinical suspicion and definitive treatment can be long, and the therapeutic decision must take into account all the risks and benefits involved.

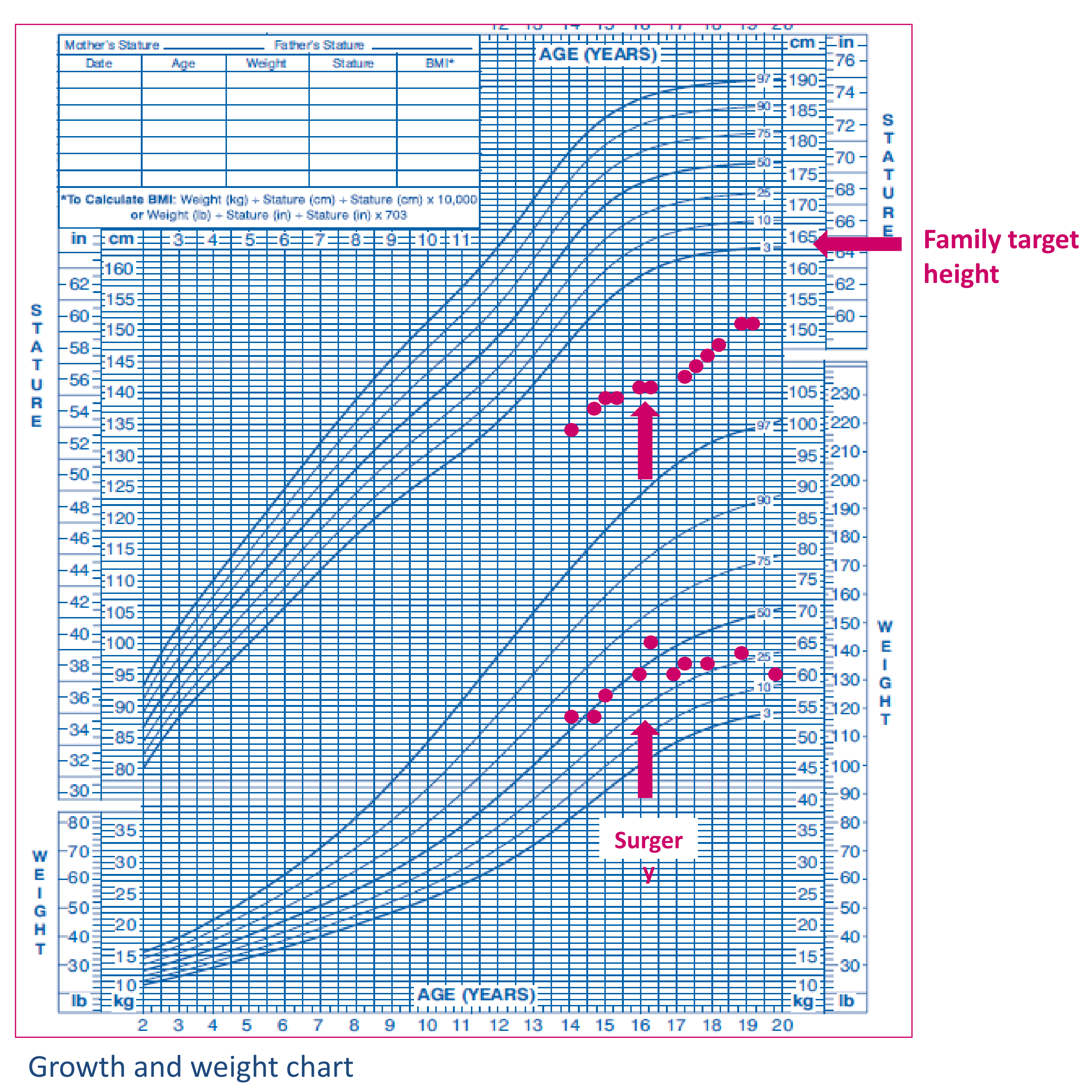

